\title{
Heart Rate Variability and the Efficacy of Biofeedback in Heroin Users with Depressive Symptoms
}

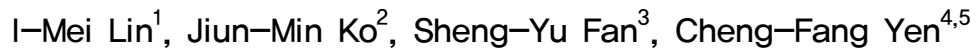 \\ 'Department of Psychology, College of Humanities and Social Science, Kaohsiung Medical University, ${ }^{2}$ Kaohsiung Drug Abuser Treatment \\ Center, Agency of Corrections, Ministry of Justice, Kaohsiung, ${ }^{3}$ Institute of Gerontology, National Cheng Kung University, Tainan, \\ ${ }^{4}$ Department of Psychiatry, Faculty of Medicine and Graduate Institute of Medicine, College of Medicine, Kaohsiung Medical University, \\ ${ }^{5}$ Department of Psychiatry, Kaohsiung Medical University Hospital, Kaohsiung, Taiwan
}

\begin{abstract}
Objective: Low heart rate variability (HRV) has been confirmed in heroin users, but the effects of heart-rate-variability-biofeedback in heroin users remain unknown. This study examined (1) correlations between depression and HRV indices; (2) group differences in HRV indices among a heroin-user group, a group with major depressive disorder but no heroin use, and healthy controls; and (3) the effects of heart-rate-variability-biofeedback on depressive symptoms, HRV indices, and respiratory rates within the heroin group.

Methods: All participants completed a depression questionnaire and underwent electrocardiogram measurements, and group differences in baseline HRV indices were examined. The heroin group underwent electrocardiogram and respiration rate measurements at baseline, during a depressive condition, and during a happiness condition, before and after which they took part in the heart-rate-variability-biofeedback program. The effects of heart-rate-variability-biofeedback on depressive symptoms, HRV indices, and respiration rates were examined.

Results: There was a negative correlation between depression and high frequency of HRV, and a positive correlation between depression and low frequency to high frequency ratio of HRV. The heroin group had a lower overall and high frequency of $H R V$, and a higher low frequency/high frequency ratio than healthy controls. The heart-rate-variability-biofeedback intervention increased HRV indices and decreased respiratory rates from pre-intervention to post-intervention.

Conclusion: Reduced parasympathetic and increased sympathetic activations were found in heroin users. Heart-rate-variabilitybiofeedback was an effective non-pharmacological intervention to restore autonomic balance.
\end{abstract}

KEY WORDS: Autonomic nervous system; Depression; Heart-rate-variability-biofeedback; Heroin.

\section{INTRODUCTION}

The prevalence of heroin use has increased in recent years. ${ }^{1-3)}$ Heroin use can cause significant psychosocial problems such as unemployment and criminal activity, as well as compromising the user's functioning. ${ }^{4}$ A high proportion of heroin users have comorbid psychiatric disorders. ${ }^{1)}$ The prevalence of major depressive episodes among heroin users has been found to be $25 \%$ to $26 \%$. ${ }^{1,5}$ Comorbidity with depression compromises the heroin user's prognosis, increasing the risk of relapsing into heroin use, and suicide. ${ }^{6}$

\footnotetext{
Received: August 20, 2015 / Revised: October 1, 2015

Accepted: October 2, 2015

Address for correspondence: Cheng-Fang Yen, MD, PhD Department of Psychiatry, Kaohsiung Medical University Hospital, 100, Tzyou 1st Road, Kaohsiung 807, Taiwan (ROC) Tel: +886-7-3121101 ext.6816, Fax: +886-7-3134761 E-mail: chfaye@cc.kmu.edu.tw
}

Previous neurobiological research has found that heroin use may damage the user's limbic system and locus ceruleus, which causes neurotransmitter changes. ${ }^{7}$ However, few studies have explored the relationships between psychophysiological responses and heroin use. Heroin withdrawal may accompany physiological responses, such as restlessness, cold sweats, cold flashes, goose bumps, an increased heart rate, an elevated temperature, an elevated pulse, a sharp elevation in blood pressure, sweating, shallow breathing, insomnia, muscle and bone pain, diarrhea, nausea, and vomiting. ${ }^{8,9)}$ Most of these heroin withdrawal symptoms involve increased sympathetic nervous system (SNS) activation, decreased parasympathetic nervous system (PNS) activation, and/or decreased cardiac vagal activity. ${ }^{10)}$ A meta-analysis of the cue-reactivity paradigm found that an increased heart rate, an increase in sweat gland activity, and a decreased skin temperature were the key physiological indicators of a

() This is an Open-Access article distributed under the terms of the Creative Commons Attribution Non-Commercial License (http://creativecommons.org/licenses/by-nc/4.0) which permits unrestricted non-commercial use, distribution, and reproduction in any medium, provided the original work is properly cited. 
craving for an addictive substance. ${ }^{11)}$ Yu et al. ${ }^{12)}$ also found that heroin-related imagery scripts and paraphernalia were able to induce cravings and changes in cardiovascular function, including an increase in systolic and diastolic blood pressure, and an increased heart rate.

The results of the previous studies described above indicate the importance of studying heart rate variability (HRV) among heroin users. HRV measures the variation in heart beat intervals, which is influenced by SNS and PNS input to the sino-atrial node of the heart. ${ }^{13)}$ Increased PNS activation (or vagal activity) increases HRV, while increased SNS activation (or sympatho-vagal imbalance) decreases HRV. ${ }^{14)}$ Regarding the interpretation of HRV indices, standard deviation of NN intervals (SDNN) and total power of HRV are indicated the overall HRV, low frequency (LF) is most likely affected both by cardiac SNS and PNS, and high frequency (HF) is an index of PNS activation. ${ }^{13,14)} \mathrm{HRV}$ is related to an individual's overall physical condition ${ }^{15,16)}$ and/or his or her mental health. ${ }^{17-19)}$ Reduced HRV has been found in heroin users ${ }^{10)}$ and patients with major depressive disorder (MDD) ${ }^{2,20)}$ Lower SDNN and HF indices have been found to be related to emotional dysregulation and social disability among patients with MDD. ${ }^{21-23)}$ Compared with healthy controls, recently relapsed heroin users show lower heart rate variance, reduced total power, and a lower HF HRV. ${ }^{10)}$ However, few studies have examined the difference in HRV indices between heroin users with depressive symptoms and patients with MDD.

Previous studies have found that methadone maintenance therapy is able to affect the HF index among heroin users, ${ }^{10,24)}$ and bring about an increase in total power and the LF index among heroin users with borderline and other personality patterns. ${ }^{24)}$ However, the effects of a non-pharmacological intervention model, such as HRVbiofeedback (HRV-BF), on HRV indices in heroin users has not been examined in detail. The protocol of HRV-BF was first described by Lehrer et al. ${ }^{25)}$ Its aim is to increase respiratory sinus arrhythmia (RSA) amplitude by breathing at the resonance frequency, which is approximately $0.1 \mathrm{~Hz}$ or six breaths per minute. ${ }^{26)}$ Lin et al. ${ }^{27)}$ modified Lehrer et al.'s ${ }^{25)}$ HRV-BF protocol and successfully applied it to patients with coronary artery disease and MDD in Taiwanese populations. ${ }^{27,28)}$ Lin et al. ${ }^{27)} \mathrm{HRV}-\mathrm{BF}$ protocol followed the Lehrer et al.' $\mathrm{s}^{25)}$ protocol by determining the individual's resonance frequency in the first session, and then adjusting the respiration rate during the HRV-BF training by gradually stepping down to reach the participant's resonance frequency, by asking participants to breathe slowly and regularly. ${ }^{27)}$ Breathing at the resonance frequency has been found to cause resonance in the cardiovascular system, thereby increasing HRV and inducing a baroreflex gain. ${ }^{16)}$ Research has confirmed the effects of HRV-BF with respect to increasing cardiac vagal activity among patients with cardiovascular disease ${ }^{15,29)}$ and with respect to decreasing depressive symptoms among patients with MDD. ${ }^{17)}$ However, the effects of HRV-BF in increasing HRV indices and decreasing depressive symptoms among heroin users still needs to be explored.

This study consisted of two parts. The aim of the first part was to compare the differences in HRV indices among heroin users with depressive symptoms, patients with MDD but no heroin use, and healthy controls. The aim of the second part was to examine the effects of HRV-BF on depressive symptoms, HRV indices, and respiratory rates among heroin users with depressive symptoms.

\section{METHODS}

\section{Study 1}

\section{Participants}

A case-control study with matching age and gender was conducted. This part of the study consisted of three groups of participants, namely heroin users with depressive symptoms (the heroin group), patients with MDD but no heroin use (the MDD group), and healthy controls. A total of 354 male addicts were invited for being screened for types of substance use and depressive symptoms by clinical psychologists at Kaohsiung Drug Abuser Treatment Center (Kaohsiung, Taiwan). Inclusion criteria were: (1) the severity and duration of heroin use fitted the diagnostic criteria of heroin dependence found in the Diagnostic and Statistical Manual of Mental Disorders, fourth edition, text revision (DSM-IV-TR) ${ }^{8}$; (2) the patient had abstained from heroin use for at least 1 month; (3) the total score of the Beck Depression Inventory-II (BDI-II) ${ }^{30)}$ was higher than 13; and (4) the patient was motivated and available to participate this study. Exclusion criteria included (1) having current or past cardiovascular disease; (2) having any severe psychiatric disorder except for MDD, such as schizophrenia or bipolar disorder; and (3) having a past history of head injury, neurological illness, or mental retardation.

The MDD group and healthy controls were selected from our data pool based on the criteria of being a male, and age matched to within 10 years of that of the heroin 
users. The MDD group was recruited from psychiatric outpatient clinics of Kaohsiung Medical University Hospital (Kaohsiung, Taiwan). The MDD group consisted of nine male patients who were diagnosed by psychiatrists as having MDD according to the criteria of DSM-IV-TR ${ }^{8)}$ and who were confirmed to have never used heroin. Nine healthy controls who visited the health center of Kaohsiung Medical University Hospital for a health checkup were recruited. Each individual's total BDI-II score was lower than 13. All participants in the MDD group and the healthy controls had no mental or physical problems as described in the exclusion criteria for the participants in the heroin group. This study was approved by the institutional review board of Kaohsiung Medical University Hospital (KMUH-IRB-2012-02-09-(I) and KMUH-IRB-990255). Written informed consent was obtained from each participant before the study started.

\section{Self-report data}

All participants filled in a questionnaire that collected demographic characteristics and depression levels. The BDI-II contains 21-item statements that assess cognitive and somatic depression during a period of 2 weeks before the study. ${ }^{30)}$ The score range of the BDI-II is from 0 to 63 . No significant depression is denoted by scores of $0-13$, mild depression by 14-19, moderate depression by $20-28$, and severe depression by $29-63 .^{30)}$

\section{Physiological measurements}

All participants were instructed not to exercise excessively and to refrain from caffeinated beverages, alcoholic beverages, and smoking for 3 hours before the psychophysiological measurements were collected. A lead II electrocardiogram (ECG) was collected for 5 minutes at baseline using the ProComp Infiniti ${ }^{\mathrm{TM}}$ system (Thought Technology Ltd., Montreal, Canada), which was installed on a laptop. A sampling rate of 2,048/second was set in order to acquire real-time interbeat intervals. The physiological signals were collected between 9:00 to 12:00 a.m. and 2:00 to 5:00 p.m. to minimize any effects of biological circadian variations. The CardiacPro Infiniti HRV analysis module (Thought Technology Ltd.) with fast Fourier transformation was used to transform the interbeat intervals into the various frequency domains of the HRV indices, namely LF power $(0.04-0.15 \mathrm{~Hz})$, HF power (0.15-0.4 Hz), and the LF/HF ratio. The time domain of the HRV index of the SDNN was also obtained.

\section{Research design and procedure}

A between-subjects design was used in this study. Participants of all three groups completed the questionnaires in a sound-attenuated and temperature-controlled room. After a 10-minute rest, an ECG sensor was placed on the participant for measuring the 5-minute period of raw ECG signals, which were transformed into the baseline HRV indices.

\section{Study 2}

\section{Participants}

The nine heroin users with depressive symptoms from Study 1 were invited to take part in the HRV-BF program.

\section{Psychophysiological measurement}

The demographic data and information related to heroin use, including the history of substance use, the age of onset of heroin use, and the number of times the participants had tried previously to quit heroin use, were collected among heroin users. The BDI-II total score and cognitive and somatic symptoms of depression were obtained at pre-intervention and post-intervention from all participants. The HRV-BF was carried out using ProComp Infiniti ${ }^{\mathrm{TM}}$ software together with $\mathrm{ECG}$ and respiration sensors, which were used to acquire real-time feedback regarding respiration rate, breathing patterns, RSA amplitude, and HRV indices. The HRV indices as the outcome variables were obtained at pre-intervention and at postintervention.

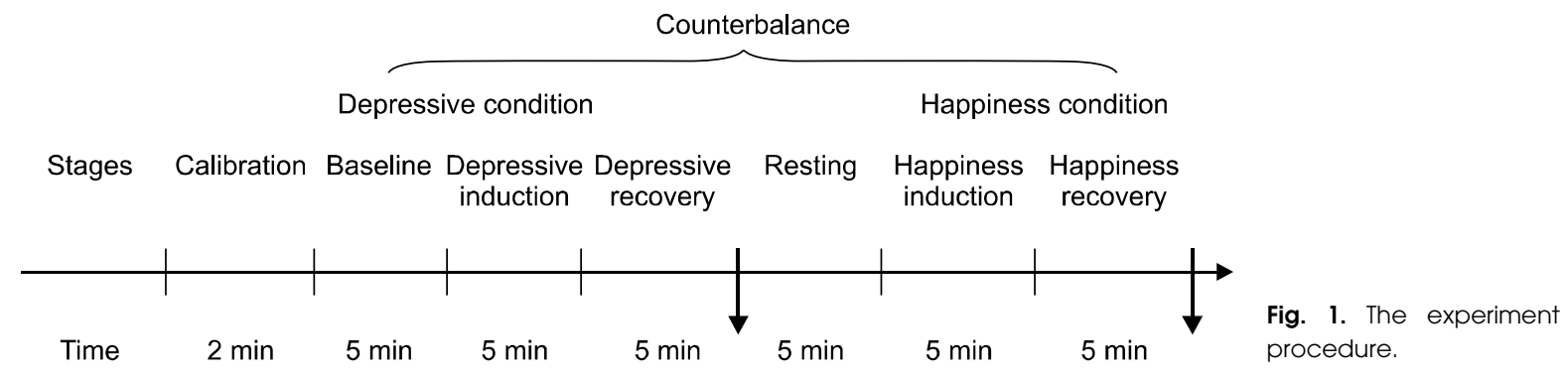




\section{Research design and procedure}

The HRV-BF protocol was based on Lin et al., ${ }^{27)}$ and included self-guided muscle relaxation, diaphragmatic breathing, paced breathing, pursed-lips breathing, and RSA-biofeedback over a 60-minute session, weekly for 6 weeks. The aims of HRV-BF were to increase LF power at $0.1 \mathrm{~Hz}$, increase RSA amplitude, decrease SNS activation, increase vagal tone or PNS activation, and restore balance of the autonomic nervous system.

A counterbalanced within-subjects experimental design was used. We quasi-randomly assigned the experimental tasks to the participants: they completed the conditions in a different order $(\mathrm{AB}$ or $\mathrm{BA}$, where $[\mathrm{A}]$ the depressive condition, and $[\mathrm{B}]$ the happiness condition). The first person completed the tasks in the order $\mathrm{AB}$, the second person in the order BA, and so on (Fig. 1). All participants were seated in a sound-attenuated and temperature-controlled room. After 2 minutes of calibration of the physiological signals, ECG measurements were obtained during 5 minutes of baseline, during a 10-minute depressive condition (5 minutes of depression induction that participants were guided to think about a depressive event and then 5 minutes of recovery from depression), and during a 10-minute happiness condition (5-minute happiness induction participants were guided to think about a happiness event and 5 minutes of recovery from happiness). There was a 5-minute break between the depressive and happiness conditions. HRV indices were compared between pre- and post-intervention.

\section{Statistics Analysis}

\section{Study 1}

The nonparametric Kruskal-Wallis test with MannWhitney U post-hoc comparisons were used to examine the group differences in age, total score, and cognitive/somatic symptoms of depression, and HRV indices across the three groups. Spearman's rank correlations were used to examine the association between depression total scores and HRV indices. The statistical analyses were performed using PASW Statistics ver. 18.0 (IBM Co., Armonk, NY, USA).

\section{Study 2}

Nonparametric Kruskal-Wallis tests were used to examine the effects of HRV-BF on the depression total score, and cognitive and somatic symptoms of depression that were measured by BDI-II; these were compared pre-inter- vention and post-intervention. The effects of HRV-BF were also examined by comparing the HRV indices and respiration rates during the various experimental conditions, pre- and post-intervention. Statistical analyses were performed using PASW Statistics ver. 18.0.

\section{RESULTS}

\section{Study 1}

A total of 90 addicts $(25.42 \%)$ with depressive symptoms were screened; however, only nine male heroin users were recruited into the heroin group based on the inclusion and exclusion criteria described above. The mean age of the participants was 40.33 years (standard deviation $[\mathrm{SD}]=8.50$, range from 29 to 54 ) for the heroin group, 33.44 years ( $\mathrm{SD}=10.55$, range from 21 to 52 ) for the MDD group, and 37.78 years ( $\mathrm{SD}=11.25$, range from 21 to 57 ) for the healthy controls. There were no significant differences in age among these three groups $\left(\chi^{2}=1.21, p>\right.$ $0.05)$. Based on the criteria suggested for the BDI-II by Beck et al., ${ }^{30)} 22.2 \%(\mathrm{n}=2)$ of the participants in the heroin group had mild depression, $55.6 \%(\mathrm{n}=5)$ had moderate depression, and $22.2 \%(\mathrm{n}=2)$ had severe depression. Twothirds $(n=6)$ of the participants in the MDD group were in remission from depression, $11.1 \%(\mathrm{n}=1)$ had mild depression, and $22.2 \%(\mathrm{n}=2)$ had severe depression. All of the healthy controls had no significant depression.

Table 1 shows the results of the Kruskal-Wallis test on depression and baseline HRV indices among the heroin group, the MDD group, and healthy controls. There were significant differences in depression total score, and cognitive and somatic symptoms of depression among the three groups $\left(\chi^{2}=16.51, p<0.001 ; \chi^{2}=13.62, p<0.001\right.$; and $\chi^{2}=12.66, p<0.01$, respectively), as well as for the HRV indices SDNN, HF, and the LF/HF ratio $\left(\chi^{2}=6.08\right.$, $p<0.05 ; \chi^{2}=9.95, p<0.01$; and $\chi^{2}=6.87, p<0.05$, respectively; Table 1). The Mann-Whitney U post-hoc comparison revealed that the heroin and MDD groups had a higher depression total score, and cognitive and somatic symptoms of depression than the healthy controls. In addition, the heroin group had lower SDNN and HF values than the healthy controls, and there was a higher LF/HF ratio in the heroin group than in healthy controls. However, there were no significant differences among the three groups in LF HRV.

Spearman's rank correlations showed a negative association between depression total score and HF HRV ( $r=$ $-0.40, p<0.05$ ), and a positive association between depression total score and the LF/HF ratio $(r=0.46, p<0.05)$ 
Table 1. The Kruskal-Wallis test on depression and baseline HRV indices among heroin, MDD, and healthy control groups ( $\mathrm{n}=27$ )

\begin{tabular}{|c|c|c|c|c|c|}
\hline & $\begin{array}{l}\text { Heroin group } \\
\qquad(n=9)\end{array}$ & $\begin{array}{l}\text { MDD group } \\
\quad(n=9)\end{array}$ & $\begin{array}{l}\text { Healthy controls } \\
\qquad(n=9)\end{array}$ & $\chi^{2}$ & $\begin{array}{l}\text { Mann-Whitney U } \\
\text { post-hoc comparison }\end{array}$ \\
\hline Age, yr (SD) & $40.33(8.50)$ & $33.44(10.55)$ & $37.78(11.25)$ & 1.21 & \\
\hline Gender, male & 9 & 9 & 9 & & \\
\hline \multicolumn{6}{|l|}{ BDI-II } \\
\hline Depression total score & 20.50 & 15.83 & 5.67 & $16.51^{\star \star \star}$ & Heroin, MDD > Healthy \\
\hline Cognitive depression & 20.33 & 15.00 & 6.67 & $13.62^{\star \star *}$ & Heroin, MDD > Healthy \\
\hline Somatic depression & 17.61 & 18.00 & 6.39 & $12.66^{* *}$ & Heroin, MDD > Healthy \\
\hline \multicolumn{6}{|l|}{ HRV indices } \\
\hline SDNN (ms) & 9.44 & 13.89 & 18.67 & $6.08^{*}$ & Healthy $>$ Heroin \\
\hline $\operatorname{LF}\left(m s^{2}\right)$ & 11.44 & 12.78 & 17.78 & 3.18 & \\
\hline $\mathrm{HF}\left(\mathrm{ms}^{2}\right)$ & 8.33 & 13.56 & 20.11 & $9.95^{\star *}$ & Healthy $>$ Heroin \\
\hline LF/HF ratio & 19.22 & 13.28 & 9.50 & $6.87^{\star}$ & Healthy $<$ Heroin \\
\hline
\end{tabular}

HRV, heart rate variability; MDD, major depressive disorder; SD, standard deviation; BDI-II, Beck Depression Inventory-II; SDNN, SD of NN intervals; LF, low frequency; HF, high frequency.

${ }^{\star} p<0.05,{ }^{* *} p<0.01,{ }^{* * *} p<0.001$.
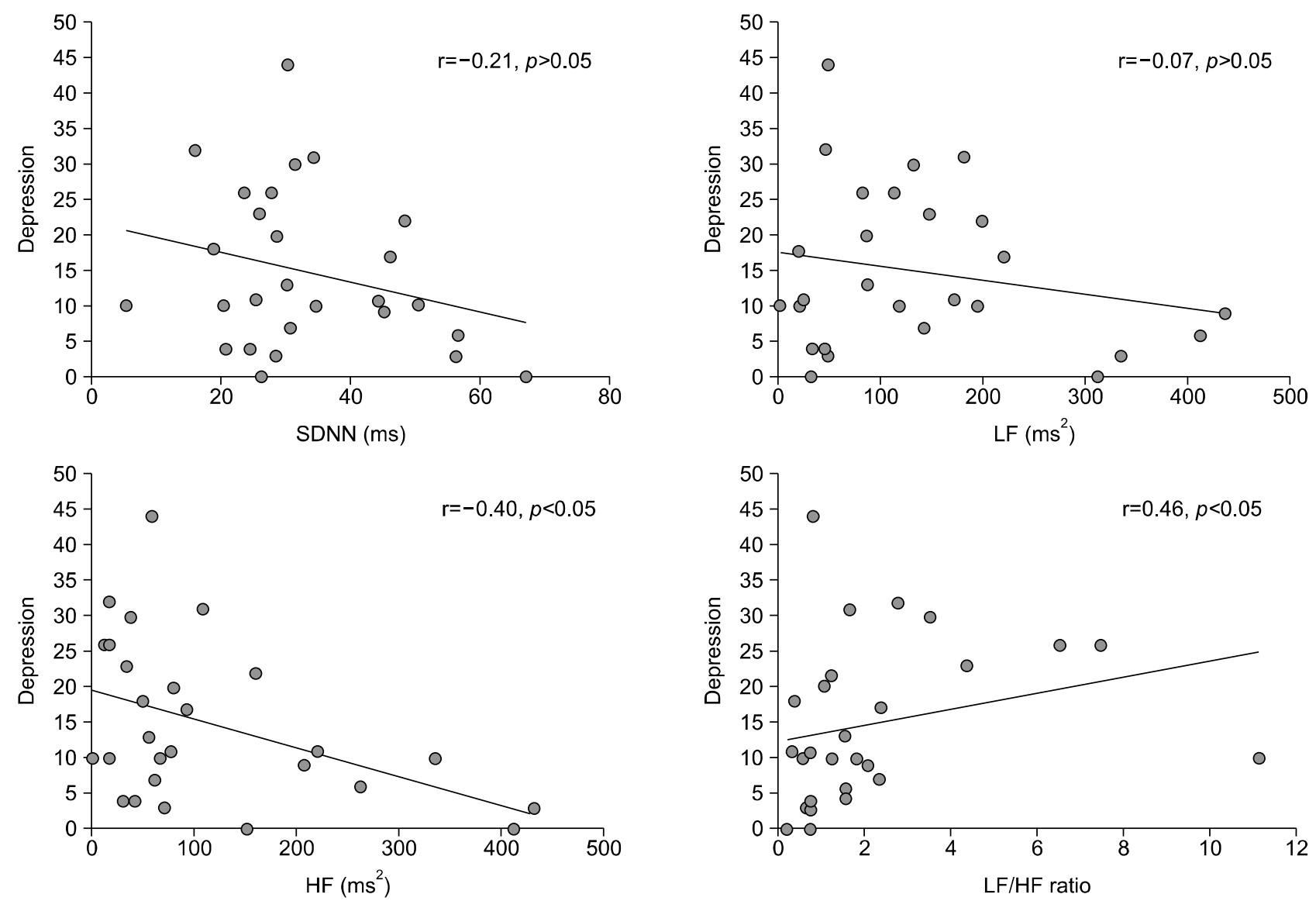

Fig. 2. The correlations between depression and the heart rate variability indices. HF, high frequency; LF, low frequency; SDNN, standard deviation of NN intervals.

for all participants; however, there was no significant association between depression total scores and SDNN or LF $(r=-0.21, p>0.05$; and $r=-0.07, p>0.05$, respectively; Fig. 2).

\section{Study 2}

Four of the heroin users combined heroin use with methamphetamine use. The range of body mass indices was 20.15 to 33.14 , with a mean of $24.09(\mathrm{SD}=4.06)$. The mean years of education was $8.56(\mathrm{SD}=4.06)$. Five of the 
Table 2. The effects of HRV-BF on HRV indices and respiration rate under various experimental conditions at pre- versus post-interventions for heroin users $(n=9)$

\begin{tabular}{|c|c|c|c|c|c|c|c|c|c|c|c|c|c|c|c|}
\hline \multirow{2}{*}{ Variable } & \multicolumn{2}{|c|}{ Baseline } & \multirow[t]{2}{*}{$z$} & \multicolumn{2}{|c|}{$\begin{array}{l}\text { Depressive } \\
\text { induction }\end{array}$} & \multirow[t]{2}{*}{$z$} & \multicolumn{2}{|c|}{$\begin{array}{c}\text { Depressive } \\
\text { recovery }\end{array}$} & \multirow[t]{2}{*}{$z$} & \multicolumn{2}{|c|}{$\begin{array}{l}\text { Happiness } \\
\text { induction }\end{array}$} & & \multicolumn{2}{|c|}{$\begin{array}{l}\text { Happiness } \\
\text { recovery }\end{array}$} & \multirow[t]{2}{*}{$z$} \\
\hline & Pre & Post & & Pre & Post & & Pre & Post & & Pre & Post & & Pre & Post & \\
\hline SDNN (ms) & $\begin{array}{c}24.97 \\
(11.82)\end{array}$ & $\begin{array}{c}33.56 \\
(12.52)\end{array}$ & -1.72 & $\begin{array}{c}28.29 \\
(10.35)\end{array}$ & $\begin{array}{c}33.38 \\
(13.39)\end{array}$ & -1.96 & $\begin{array}{l}29.10 \\
(9.62)\end{array}$ & $\begin{array}{c}47.99 \\
(19.51)\end{array}$ & $-2.38^{*}$ & $\begin{array}{l}25.53 \\
(9.91)\end{array}$ & $\begin{array}{c}32.10 \\
(11.16)\end{array}$ & -1.86 & $\begin{array}{c}28.19 \\
(12.81)\end{array}$ & $\begin{array}{c}39.72 \\
(14.30)\end{array}$ & -1.52 \\
\hline $\operatorname{LF}\left(\mathrm{ms}^{2}\right)$ & $\begin{array}{c}91.50 \\
(63.27)\end{array}$ & $\begin{array}{c}227.88 \\
(256.25)\end{array}$ & -1.13 & $\begin{array}{c}88.65 \\
(70.83)\end{array}$ & $\begin{array}{c}179.85 \\
(221.90)\end{array}$ & -1.01 & $\begin{array}{c}101.35 \\
(122.04)\end{array}$ & $\begin{array}{c}575.96 \\
(755.21)\end{array}$ & $-2.24^{*}$ & $\begin{array}{c}62.65 \\
(58.16)\end{array}$ & $\begin{array}{c}96.83 \\
(63.98)\end{array}$ & -1.35 & $\begin{array}{l}103.18 \\
(85.57)\end{array}$ & $\begin{array}{c}356.15 \\
(407.20)\end{array}$ & -1.01 \\
\hline $\mathrm{HF}\left(m s^{2}\right)$ & $\begin{array}{c}44.76 \\
(49.03)\end{array}$ & $\begin{array}{c}75.00 \\
(57.53)\end{array}$ & $-2.67^{\star \star}$ & $\begin{array}{c}42.88 \\
(35.06)\end{array}$ & $\begin{array}{c}92.00 \\
(86.48)\end{array}$ & $-2.07^{\star}$ & $\begin{array}{c}32.96 \\
(20.08)\end{array}$ & $\begin{array}{c}90.14 \\
(62.05)\end{array}$ & $-1.96^{*}$ & $\begin{array}{c}42.76 \\
(21.71)\end{array}$ & $\begin{array}{c}84.49 \\
(72.07)\end{array}$ & -1.18 & $\begin{array}{c}41.84 \\
(30.26)\end{array}$ & $\begin{array}{c}73.47 \\
(62.52)\end{array}$ & -0.51 \\
\hline $\begin{array}{l}\text { Respiration } \\
\text { rate }\end{array}$ & $\begin{array}{l}15.65 \\
(3.14)\end{array}$ & $\begin{array}{l}13.16 \\
(4.86)\end{array}$ & $-2.67^{\star *}$ & $\begin{array}{l}18.29 \\
(3.63)\end{array}$ & $\begin{array}{l}14.58 \\
(4.59)\end{array}$ & $-2.43^{*}$ & $\begin{array}{l}18.28 \\
(3.92)\end{array}$ & $\begin{array}{l}12.04 \\
(3.89)\end{array}$ & $-2.55^{\star}$ & $\begin{array}{l}18.54 \\
(3.03)\end{array}$ & $\begin{array}{l}13.13 \\
(2.81)\end{array}$ & $-2.52^{\star}$ & $\begin{array}{l}19.23 \\
(3.65)\end{array}$ & $\begin{array}{l}12.93 \\
(4.88)\end{array}$ & $-2.24^{*}$ \\
\hline
\end{tabular}

HRV, heart rate variability; SDNN, standard deviation of NN intervals; LF, low frequency; HF, high frequency. ${ }^{*} p<0.05,{ }^{* *} p<0.01$.
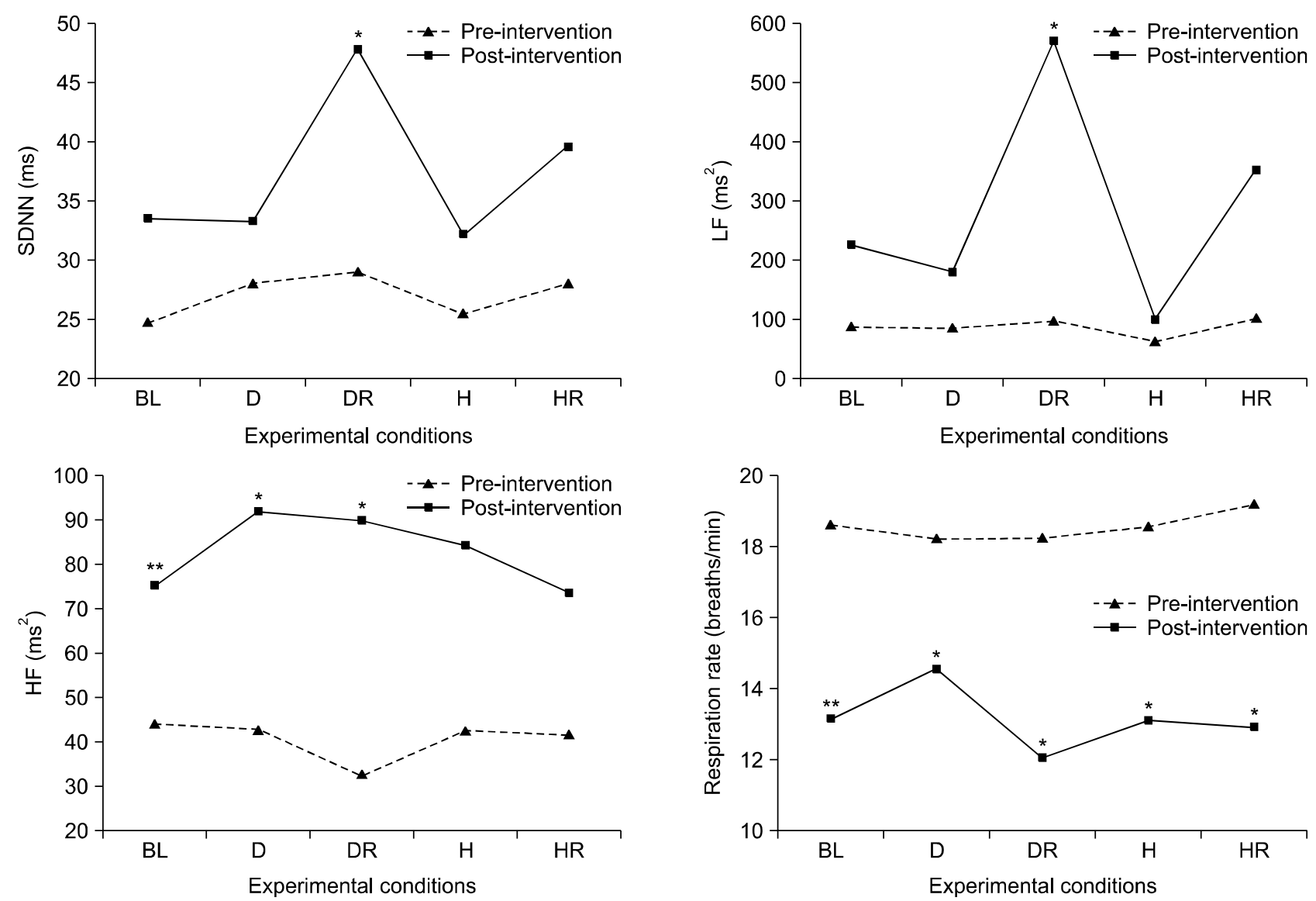

Fig. 3. The heart rate variability indices (SDNN, LF, and HF) and respiration rate under various experimental conditions at pre- versus post-intervention. SDNN, standard deviation of NN intervals; LF, low frequency; HF, high frequency; BL, baseline; $D$, depressive induction; $D R$, depressive recovery; $H$, happiness induction; $H R$, happiness recovery.

group were single $(55.60 \%)$ and four were divorced or separated $(44.40 \%)$. The mean age of initial heroin use was 20.33 years $(\mathrm{SD}=4.44$, range from 14 to 28$)$. The average number of attempts to quit heroin use was 2.33 ( $\mathrm{SD}=1.41$, range from 1 to 5 times).

Regarding the effects of HRV-BF on depressive symp- toms, the depression total score decreased from 23.00 pre-intervention to 18.33 post-intervention, and cognitive depression decreased from 19.00 to 14.33 . However, the Kruskal-Wallis test showed that there were no significant differences in depression total score, and cognitive and somatic symptoms of depression between pre- versus 
post-intervention $(z=-1.61, p>0.05 ; z=-1.72, p>0.05$; and $z=0.00, p>0.05$, respectively).

Table 2 and Figure 3 show the pre- versus post-intervention comparisons of the HRV indices and respiration rates for the various experimental conditions, using the Kruskal-Wallis test. There were significant increases in SDNN, LF, and HF for depressive recovery from pre-intervention to post-intervention (SDNN: $z=-2.38, p<0.05$; LF: $z=-2.24, p<0.05$; and HF: $z=-1.96, p<0.05$, respectively). The HF HRV for baseline $(z=-2.67, p$ $<0.01)$ and depressive induction $(z=-2.07, p<0.05)$ also increased from pre- to post-intervention. In addition, the average pre-intervention respiration rate was 18.60 breaths per minute, which reduced to 13.17 breaths per minute post-intervention. Respiration rates decreased significantly pre- to post-intervention for the five experimental conditions $(z=-2.67, p<0.01 ; z=-2.43, p$ $<0.05 ; z=-2.55, p<0.05 ; z=-2.52, p<0.05 ; z=-2.24$, $p<0.05$, respectively).

\section{DISCUSSION}

The first part of this study examined group differences in HRV indices between a heroin user group, a MDD group, and healthy controls. The heroin group had lower SDNN and HF HRV indices than healthy controls. In addition, our result also confirmed that the heroin group had a higher LF/HF ratio than the healthy controls, which is consistent with the findings of Chang et al. ${ }^{10)}$ for heroin users who were receiving methadone maintenance therapy. These results indicate that the heroin group had decreased PNS activation and increased SNS activation. However, we did not find a group difference in HRV indices between the heroin group and the MDD group. It has been shown that patients with MDD have decreased SDNN and $\mathrm{HF}^{31)}$ which may account for this lack of significant differences.

In addition, we also found a negative correction between depression total score and HF HRV, and a positive correlation between depression total score and the LF/HF ratio. This result is consistent with the findings of Agelink et al., ${ }^{32)}$ and indicates that PNS withdrawal occurs in patients with MDD. The polyvagal theory ${ }^{33)}$ emphasizes the role of cardiovascular physiology in social behavior whereby the vagal brake regulates sympathetic activation. Individuals with substance addiction may use addictive drugs to reduce this negative effect or to modulate interpersonal conflicts. As a result, continued drug addiction may impair the vagal activity of drug users. ${ }^{34,35)}$
Regarding the effects of HRV-BF on HRV indices in heroin users, we found a significant increase in HRV indices from pre- to post-intervention, especially for SDNN, $\mathrm{LF}$, and $\mathrm{HF}$ under recovery from depression, and HF under baseline and depressive induction. The increased SDNN and LF are consistent with the results of a previous study on the effects of HRV-BF among patients with MDD. ${ }^{17,28)}$ The effects of HRV-BF with respect to increasing HF observed in this study are also consistent with findings in patients with coronary artery disease. ${ }^{36,37)}$ The present study found that heroin users slowed their respiration rates from 18.60 to 13.17 breaths per minute after HRV-BF intervention. Slowing down the respiration rate is known to result in a significant increase in PNS activation. ${ }^{23)}$ The findings of this study support the hypothesis that HRV-BF is an effective non-pharmacological intervention that restores parasympathetic activation among heroin users.

It is noteworthy that we found no significant increase in HRV indices for the happiness induction and happiness recovery conditions, from pre- to post-intervention. This result is consistent with a prior study wherein heroin users had a greater emotional response to neutral images than to pleasant images. ${ }^{38)}$ It is possible that this effect in heroin users is related to both impaired negative emotional processing $^{39)}$ and to the compromising effect of heroin on the brain, such as an effect on amygdala activity. ${ }^{40)}$ Consequently, it may be difficult for heroin users to experience a positive emotion. The amygdala is thought to play an important role in processing anxiety and depression related to drug dependence via negative reinforcement. ${ }^{40)}$ This may explain why we were unable to find a significant improvement in HRV indices for happiness induction and happiness recovery, comparing pre- to post-intervention. Overall, heroin users have the difficulty to experience the positive emotion may relate to a reduction in PNS inhibition of SNS activity, blocking vagal inhibitory processes and baroreflex feedback. ${ }^{33)}$

There were several limitations of our investigation that deserve attention. First, the small sample size of our preliminary study limits wide generalization of our results. Although we screened 90 addicts from 354 clients whose depression total score exceeded 13 , only nine heroin users participated and completed the 6-week HRV-BF. Numerous reasons may have limited participation in this study, such as the participant not using heroin, having a routine activity or other treatment program that was a contraindication for study participation, moving to another jail such that they could not complete the 6-week program, and a lack of motivation to participate in the study. Second, the diag- 
nosis of MDD was not confirmed by psychiatrists. Only one heroin user reported a previous MDD diagnosis by psychiatrist and a prior prescription for antidepressants. Third, there was no control group in Study 2, which limits the interpretation of the effects of HRV-BF.

In conclusion, this study found that heroin users with depressive symptoms show reduced PNS activation and increased SNS activation. HRV-BF was found to be an effective non-pharmacological intervention that was able to restore the autonomic balance of heroin users. The HRV-BF protocol with breathing training thus may be an intervention strategy for heroin users in order to improve their cardiac autonomic balance.

\section{Acknowledgments}

This study was partially supported by grant NSC1012314-B-037-057-MY3 awarded by the National Science Council, Taiwan (ROC). We gratefully thank Yu-Ting Chen, Che-Cheng Chu, Hui-Ping Chan, and Hsueh-Chen $\mathrm{Lu}$ at Kaohsiung Medical University for assistance with the experimental procedures. We also thank Yao-Chien Chen at Kaohsiung Drug Abuse Treatment Center, Agency of Correction, Ministry of Justice.

\section{REFERENCES}

1. Fan CY, Tan HK, Chien IC, Chou SY. Prevalence of psychiatric disorders among heroin users who received methadone maintenance therapy in Taiwan. Am J Addict 2014;23:249-256

2. King R, Bird SM, Overstall AM, Hay G, Hutchinson SJ. Estimating prevalence of injecting drug users and associated heroin related death rates in England by using regional data and incorporating prior information. J Royal Stat Soc Ser A 2014;177:209-236.

3. McNeely J, Gourevitch MN, Paone D, Shah S, Wright S, Heller D. Estimating the prevalence of illicit opioid use in New York City using multiple data sources. BMC Public Health 2012;12:443.

4. Marel C, Mills KL, Darke S, Ross J, Slade T, Burns L, et al. Static and dynamic predictors of criminal involvement among people with heroin dependence: findings from a 3-year longitudinal study. Drug Alcohol Depend 2013; 133:600-606.

5. Kuhlmann S, Piel M, Wolf OT. Impaired memory retrieval after psychosocial stress in healthy young men. J Neurosci 2005;25:2977-2982.

6. Chen VC, Lin TY, Lee CT, Lai TJ, Chen H, Ferri CP, et al. Suicide attempts prior to starting methadone maintenance treatment in Taiwan. Drug Alcohol Depend 2010;109:139-143.

7. Grüsser SM, Thalemann CN, Platz W, Gölz J, Partecke G. $A$ new approach to preventing relapse in opiate addicts: a psychometric evaluation. Biol Psychol 2006;71:231-235.

8. American Psychiatric Association. Diagnostic and Statistical Manual of Mental Disorders, fourth edition, text revision (4th ed-TR). Washington, DC:American Psychiatric Association;2000.
9. Grella CE, Lovinger K. Gender differences in physical and mental health outcomes among an aging cohort of individuals with a history of heroin dependence. Addict Behav 2012;37:306-312.

10. Chang LR, Lin YH, Kuo TB, Ho YC, Chen SH, Wu Chang $\mathrm{HC}$, et al. Cardiac autonomic modulation during methadone therapy among heroin users: a pilot study. Prog Neuropsychopharmacol Biol Psychiatry 2012;37:188-193.

11. Carter BL, Tiffany ST. Meta-analysis of cue-reactivity in addiction research. Addiction 1999;94:327-340.

12. Yu J, Zhang S, Epstein DH, Fang Y, Shi J, Qin H, et al. Gender and stimulus difference in cue-induced responses in abstinent heroin users. Pharmacol Biochem Behav 2007;86:485-492.

13. Karim N, Hasan JA, Ali SS. Heart rate variability-a review. Aust J Basic Appl Sci 2011;7:71-77.

14. Task Force of the European Society of Cardiology the North American Society of Pacing Electrophysiology. Heart rate variability: Standards of measurement, physiological interpretation, and clinical use. Circulation 1996;93:10431065.

15. Del Pozo JM, Gevirtz RN, Scher B, Guarneri E. Biofeedback treatment increases heart rate variability in patients with known coronary artery disease. Am Heart J 2004;147:E11.

16. Lehrer P, Vaschillo E, Lu SE, Eckberg D, Vaschillo B, Scardella A, et al. Heart rate variability biofeedback: effects of age on heart rate variability, baroreflex gain, and asthma. Chest 2006;129:278-284.

17. Karavidas MK, Lehrer PM, Vaschillo E, Vaschillo B, Marin $\mathrm{H}$, Buyske S, et al. Preliminary results of an open label study of heart rate variability biofeedback for the treatment of major depression. Appl Psychophysiol Biofeedback 2007;32:19-30.

18. Kemp AH, Quintana DS, Gray MA. Is heart rate variability reduced in depression without cardiovascular disease? Biol Psychiatry 2011;69:e3-e4.

19. Tan G, Dao TK, Farmer L, Sutherland RJ, Gevirtz R. Heart rate variability $(H R V)$ and posttraumatic stress disorder (PTSD): a pilot study. Appl Psychophysiol Biofeedback 2011;36:27-35.

20. Licht CM, de Geus EJ, Zitman FG, Hoogendijk WJ, van Dyck R, Penninx BW. Association between major depressive disorder and heart rate variability in the Netherlands Study of Depression and Anxiety (NESDA). Arch Gen Psychiatry 2008;65:1358-1367.

21. Cyranowski JM, Hofkens TL, Swartz HA, Salomon K, Gianaros PJ. Cardiac vagal control in nonmedicated depressed women and nondepressed controls: impact of depression status, lifetime trauma history, and respiratory factors. Psychosom Med 2011;73:336-343.

22. Rottenberg J, Chambers AS, Allen JJ, Manber R. Cardiac vagal control in the severity and course of depression: the importance of symptomatic heterogeneity. $J$ Affect Disord 2007; 103:173-179.

23. Rottenberg J, Clift A, Bolden S, Salomon K. RSA fluctuation in major depressive disorder. Psychophysiology 2007;44: 450-458.

24. Huang WL, Lin YH, Kuo TB, Chang LR, Chen YZ, Yang CC. Methadone-mediated autonomic functioning of male patients with heroin dependence: the influence of borderline personality pattern. PLoS One 2012;7:e37464.

25. Lehrer PM, Vaschillo E, Vaschillo B. Resonant frequency biofeedback training to increase cardiac variability: rationale and manual for training. Appl Psychophysiol Biofeedback 2000;25:177-191. 
26. Vaschillo E, Lehrer P, Rishe N, Konstantinov M. Heart rate variability biofeedback as a method for assessing baroreflex function: a preliminary study of resonance in the cardiovascular system. Appl Psychophysiol Biofeedback 2002;27: 1-27.

27. Lin IM, Fan SY, Lu HC, Lin TH, Chu CS, Kuo HF, et al. Randomized controlled trial of heart rate variability biofeedback in cardiac autonomic and hostility among patients with coronary artery disease. Behav Res Ther 2015, 70:38-46.

28. Lin IM, Fan SY, Tang ZC, Peper E. Heart rate variability biofeedback improves depression and sleep quality in patients with major depression disorder. Paper presented at the poster session of the 44th annual meeting of Association for Applied Psychophysiology and Biofeedback (AAPB). 2013 Mar 14-16; Portland, OR. Portland:AABP, 2013.

29. Swanson KS, Gevirtz RN, Brown M, Spira J, Guarneri E, Stoletniy L. The effect of biofeedback on function in patients with heart failure. Appl Psychophysiol Biofeedback 2009; 34:71-91.

30. Beck AT, Steer RA, Brown GK. Beck depression inventory manual. 2nd ed. San Antonio, TX:Pyschologcial Corporation; 1996.

31. Kemp AH, Quintana DS, Gray MA, Felmingham KL, Brown K, Gatt JM. Impact of depression and antidepressant treatment on heart rate variability: a review and metaanalysis. Biol Psychiatry 2010;67:1067-1074.

32. Agelink MW, Boz C, Ullrich H, Andrich J. Relationship between major depression and heart rate variability. Clinical consequences and implications for antidepressive treatment. Psychiatry Res 2002;113:139-149.

33. Porges SW. The polyvagal theory: Neurophysiological foundations of emotions, attachment, communication, and self-regulation. New York:W. W. Norton \& Company;2011.

34. Grant VV, Stewart SH, Mohr CD. Coping-anxiety and copingdepression motives predict different daily mood-drinking relationships. Psychol Addict Behav 2009;23:226-237.

35. Koob GF, Le Moal M. Drug addiction, dysregulation of reward, and allostasis. Neuropsychopharmacology 2001; 24:97-129.

36. Cowan MJ, Pike KC, Budzynski HK. Psychosocial nursing therapy following sudden cardiac arrest: impact on two-year survival. Nurs Res 2001;50:68-76.

37. Nolan RP, Kamath MV, Floras JS, Stanley J, Pang C, Picton $\mathrm{P}$, et al. Heart rate variability biofeedback as a behavioral neurocardiac intervention to enhance vagal heart rate control. Am Heart J 2005; 149:1137.

38. Aguilar de Arcos F, Verdejo-García A, Peralta-Ramírez MI, Sánchez-Barrera M, Pérez-García M. Experience of emotions in substance abusers exposed to images containing neutral, positive, and negative affective stimuli. Drug Alcohol Depend 2005:78:159-167.

39. Blum J, Gerber H, Gerhard U, Schmid O, Petitjean S, Riecher-Rössler A, et al. Acute effects of heroin on emotions in heroin-dependent patients. Am J Addict 2013;22:598-604.

40. Schmidt A, Borgwardt S, Gerber H, Wiesbeck GA, Schmid $\mathrm{O}$, Riecher-Rössler A, et al. Acute effects of heroin on negative emotional processing: relation of amygdala activity and stress-related responses. Biol Psychiatry 2014;76:289-296. 